\title{
The association between the TP53 Arg72Pro polymorphism and colorectal cancer: An updated meta-analysis based on 32 studies
}

\author{
Xin Tian ${ }^{1}$, Shundong Dai ${ }^{2,3}$, Jing Sun ${ }^{4}$, Shenyi Jiang ${ }^{5}$, Youhong Jiang ${ }^{1}$ \\ ${ }^{1}$ Molecular Oncology Laboratory of Cancer Research Institute, The First Affiliated Hospital of China Medical University, \\ Shenyang, 110001, PR China \\ ${ }^{2}$ Department of Pathology, The First Affiliated Hospital and College of Basic Medical Sciences of China Medical University, \\ Shenyang, 110001, PR China \\ ${ }^{3}$ Institute of Pathology and Pathophysiology, Shenyang, 110001, PR China \\ ${ }^{4}$ Department of Immunology and Biotherapy, Liaoning Cancer Hospital and Institute, Shenyang, 110042, PR China \\ ${ }^{5}$ Department of Rheumatology, The First Affiliated Hospital of China Medical University, Shenyang, 110001, PR China \\ Correspondence to: Xin Tian, email: tianxin@cmulh.com
}

Keywords: TP53, colorectal cancer, polymorphism, meta-analysis

Received: July 05, $2016 \quad$ Accepted: November 08, 2016

Published: November 25, 2016

\section{ABSTRACT}

Several previous studies evaluated the association between the Arg72Pro (rs1042522) polymorphism in the TP53 tumor suppressor gene and colorectal cancer (CRC). However, the results are conflicting. This meta-analysis aimed to shed new light on the precise association between TP53 variants and CRC. We analyzed 32 published case-control studies involving 8,586 cases and 10,275 controls using crude odd ratios (ORs) with $95 \%$ confidence intervals (CIs). The meta-analysis was performed using a fixed-effect or random-effects model, as appropriate. We found that the TP53 Arg72Pro polymorphism was not significantly associated with CRC risk in the overall population. However, subgroup analysis based on ethnicity revealed an increased risk of CRC among Asians (CC vs. GC+GG: OR=1.22, 95\% CI: 1.02-1.45), and similar results were found for rectal cancer (CC vs. GC+GG: OR=1.34, 95\% CI: 1.1201.62). These results suggest that the TP53 Arg72Pro polymorphism CC genotype may contribute to an increased risk of CRC, especially for rectal cancer and among Asians.

\section{INTRODUCTION}

Colorectal cancer (CRC) is the third most commonly diagnosed cancer in males and the second most commonly diagnosed cancer in females. CRC is also the leading cause of cancer-related death in the Western world, and has exhibited a striking rise in incidence in Asian countries [1-3]. The etiology of CRC is multifactorial, though it is widely accepted that CRC can be caused by an accumulation of mutations in various genes [4]. The identification of CRC-related genes may help facilitate the early diagnosis, prevention and treatment of the disease [5].

The TP53 tumor suppressor gene, which is located on chromosome $17 \mathrm{p} 13$, is one of the most frequently mutated in human carcinogenesis [6]. The encoded TP53 protein is a key mediator in many cellular processes, including cell cycle arrest, apoptosis, senescence, DNA repair, and changes in metabolism [7]. Consequently, TP53 mutations may result in a loss of the protein's tumor suppressor function and thus contribute to the development of malignant tumors. The common TP53 Arg72Pro polymorphism (rs 1042522) at codon 72 of exon 4 is the most studied polymorphism in cancer [8]. The guanine to cytosine $(\mathrm{G}>\mathrm{C})$ nucleotide exchange associated with this polymorphism leads to a nonsynonymous amino acid change from arginine to proline. The $72 \mathrm{Arg}$ variant of TP53 exhibits enhanced ability to localize to the mitochondria and induce apoptosis, whereas the 72Pro variant more efficiently induces cell cycle arrest [9].

Several studies have been conducted to investigate the association between the TP53 Arg72Pro polymorphism and CRC. However, the results are inconsistent and conflicting. The present meta-analysis was performed to provide a more precise estimation of this association. 


\section{RESULTS}

\section{Study characteristics}

Our search strategy yielded a total of 545 records, which were screened to identify original research articles pertaining to TP53 and CRC. The literature search and detailed selection procedures are summarized in Figure 1. After the primary screening, the full text of 40 articles was retrieved for further assessment [10-49]. Ten of those articles were then excluded from further analysis: 6 were not case-control studies [40-45], 1 was based on duplicate data from another eligible study [46], and 3 reported a genotype distribution among the controls that was not in Hardy-Weinberg equilibrium (HWE) [47-49]. Two of the articles reported 2 studies each [19, 24]. Thus, 1 study in each of 28 articles and 2 studies in each of 2 articles, adds up to a total of 32 studies in 30 articles [10-39]. In these 32 studies that conformed to our inclusion criteria, there were 8586 CRC cases and 10275 controls. Fourteen studies involved Asian participants, 12 involved Caucasians, and 6 involved mixed populations. The population characteristics of the included studies are shown in Table 1.

\section{Meta-analysis results}

We assessed the association between the TP53 Arg72Pro polymorphism and CRC susceptibility by calculating an odds ratio (OR) and its $95 \%$ confidence interval (CI) under the following four genetic models: the allele model (C vs. G), the homozygote model ( $\mathrm{CC}$ vs. $\mathrm{GG})$, the dominant model $(\mathrm{CC}+\mathrm{GC}$ vs. $\mathrm{GG})$, and the recessive model (CC vs. GC+GG). A summary of our meta-analysis of the association between the TP53 Arg72Pro polymorphism and CRC is shown in Table 2. Overall, we observed no significant associations in any of the genetic models (C vs. G: OR $=1.02,95 \%$ CI 0.94 1.10; $\mathrm{CC}$ vs. GG: $\mathrm{OR}=1.06,95 \% \mathrm{CI} 0.90-1.25 ; \mathrm{CC}+\mathrm{GC}$ vs. GG: $\mathrm{OR}=1.01,95 \% \mathrm{CI} 0.91-1.11$; $\mathrm{CC}$ vs. $\mathrm{GC}+\mathrm{GG}$ : $\mathrm{OR}=1.09$, 95\%CI 0.95-1.24) (Figure 2). Further subgroup analyses were conducted to assess the effects of potential confounding factors. There was no evidence for an association between TP53 Arg72Pro polymorphism and CRC risk in subgroup analyses based on the source of the controls or the type of CRC (Table 2). However, when stratified based on tumor location, we found that the $\mathrm{CC}$ genotype increased the risk of rectal cancer (CC vs. $\mathrm{GC}+\mathrm{GG}$ : $\mathrm{OR}=1.34,95 \% \mathrm{CI} 1.12-1.62)$, but did not alter the risk of colon cancer ( $\mathrm{CC}$ vs. $\mathrm{GC}+\mathrm{GG}$ : $\mathrm{OR}=1.14$, 95\% CI 0.94-1.39). When the data for rectal cancer were stratified based on ethnicity, no significant associations were observed between TP53 Arg72Pro polymorphism and $\mathrm{CRC}$ risk. Similarly, no associations were found for colon cancer (Table 2). Nonetheless, after stratification based on ethnicity, a significant risk was observed among subjects in Asian populations who carried the $\mathrm{CC}$ genotype ( $\mathrm{CC}$ vs. $\mathrm{GC}+\mathrm{GG}$ : $\mathrm{OR}=1.22,95 \% \mathrm{CI} 1.02$ 1.45), whereas no risk was observed in Caucasian and mixed populations $(\mathrm{CC}$ vs. $\mathrm{GC}+\mathrm{GG}$ : $\mathrm{OR}=0.94,95 \% \mathrm{CI}$ $0.76-1.16$ and $\mathrm{OR}=0.82,95 \% \mathrm{CI} 0.5-1.16$, respectively). Subgroup analyses based on ethnicity revealed no significant association between TP53 Arg72Pro polymorphism and CRC risk in Caucasian and Mixed populations.

\section{Publication bias and sensitivity analysis}

We used Begg's funnel plot and Egger's test to assess the publication bias of the published articles. The symmetrical funnel plot for the allele model shown in Figure 3 suggests the findings of our meta-analysis were not affected by publication bias. The Egger's test results also did not suggest the existence of publication bias, as indicated by $P$ values greater than 0.05 ( $P=0.098$ for the allele model). The influence of each individual study on the pooled OR was assessed by performing the analysis while deleting one study at a time. Because the OR was not significantly influenced by omitting any single study (data not shown), we conclude our data are relatively stable and credible.

\section{DISCUSSION}

The mechanisms that underlie the development of CRC are complex, and both environmental and genetic factors play important roles in the occurrence and progression of this disease [50]. TP53 is crucial for proper control of gene transcription, DNA synthesis and repair, cell cycle arrest, senescence and apoptosis. Mutations in TP53 can disrupt these functions, leading to genetic instability and the progression to cancer.

In this meta-analysis, we found that the TP53 Arg72Pro polymorphism was not associated with CRC in patients stratified based on type of CRC, genotype method or source of controls. When stratified based on ethnicity, there was a positive association between the TP53 Arg72Pro polymorphism and CRC risk in Asian populations, but not Caucasian or mixed populations. These differences may reflect differences in genetic background and/or environmental factors. The Arg72 variant of the TP53 Arg72Pro polymorphism is more efficient with respect to mitochondrial localization than the Pro72 variant and has a stronger capacity to induce apoptosis [51]. Researchers observed that the Arg 72 form induced apoptosis with faster kinetics than did the Pro72 variant [52]. The greater apoptotic potential of the Arg72 protein stems from the greater interaction of this protein 
with MDM2, which facilitates nuclear export [53]. The two polymorphic variants of TP53 are functionally distinct, and these differences may influence cancer risk or treatment. Our result is does not confirm the findings of 2 earlier meta-analyses [54, 55]. These differences may be the result of the rigid inclusion criteria of our study. We excluded two studies with control genotypic distributions that deviated from the $\operatorname{HWE}[47,48]$ and

\section{Potential relevant articles identified through database searching or other sources $(n=545)$.}

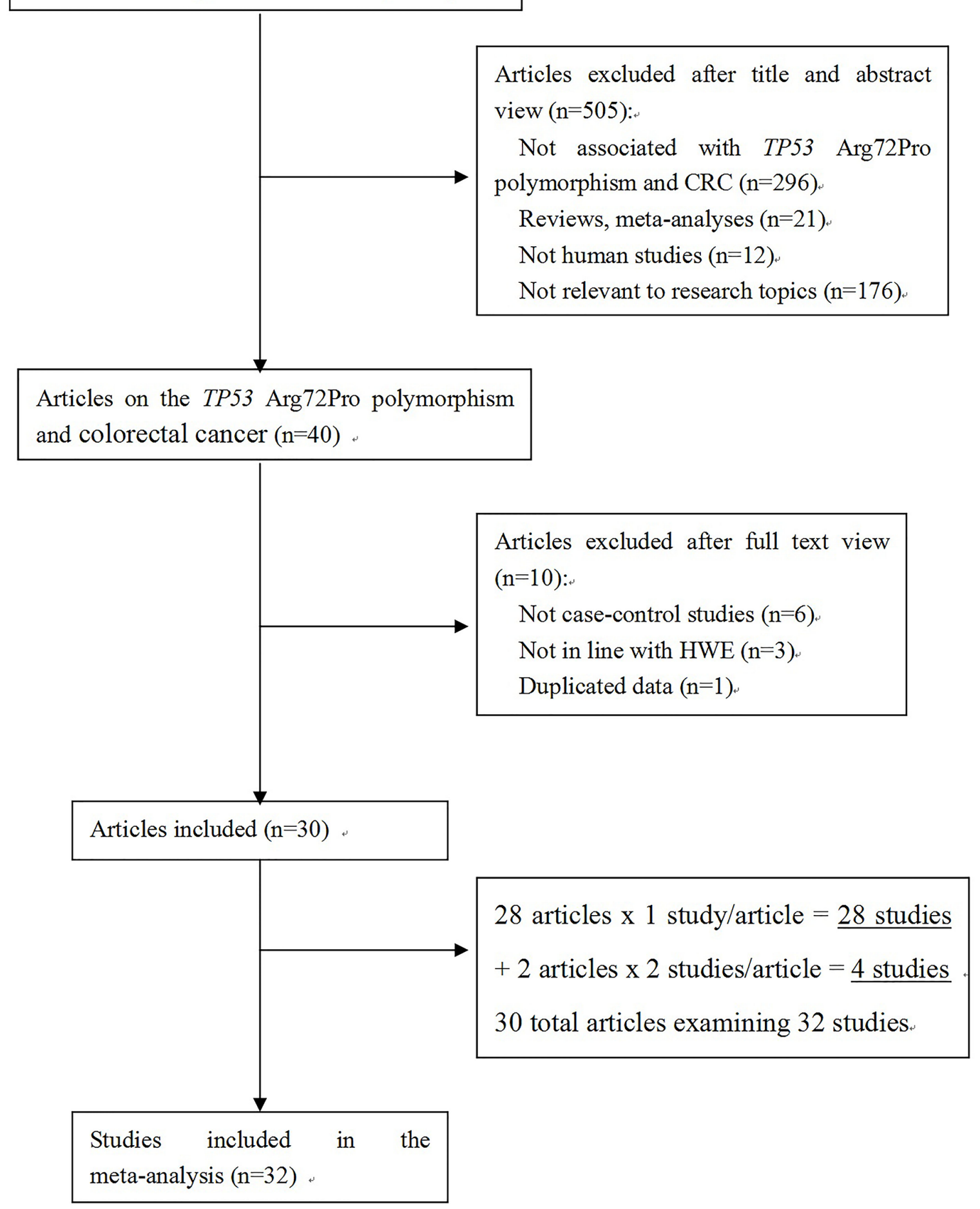

Figure 1: Flow chart of study selection process. 
Table 1: Characteristics of the individual studies included in the meta-analysis

\begin{tabular}{|c|c|c|c|c|c|c|c|c|c|c|c|c|c|}
\hline \multirow[t]{2}{*}{ First author } & \multirow[t]{2}{*}{ Year } & \multirow[t]{2}{*}{ Country } & \multirow[t]{2}{*}{ Ethnicity } & \multirow{2}{*}{$\begin{array}{c}\text { Source of } \\
\text { control }\end{array}$} & \multirow{2}{*}{$\begin{array}{c}\text { Type of } \\
\text { CRC }\end{array}$} & \multicolumn{3}{|c|}{ Cases } & \multicolumn{3}{|c|}{ Controls } & \multirow[t]{2}{*}{ HWE } & \multirow[t]{2}{*}{ Methods } \\
\hline & & & & & & GG & GC & $\mathrm{CC}$ & GG & GC & $\mathbf{C C}$ & & \\
\hline Olschwang ${ }^{10}$ & 1991 & France & Caucasian & Population-based & Sporadic & 32 & 34 & 5 & 49 & 52 & 14 & 0.97 & PCR-RFLP \\
\hline Kawajiri $^{11}$ & 1993 & Japan & Asian & Population-based & Sporadic & 36 & 32 & 16 & 144 & 165 & 38 & 0.36 & Allele specific PCR \\
\hline Murata $^{12}$ & 1996 & Japan & Asian & Hospital-based & Sporadic & 46 & 55 & 14 & 53 & 76 & 23 & 0.62 & Allele specific PCR \\
\hline Wang $^{13}$ & 1999 & China & Asian & Hospital-based & Sporadic & 18 & 33 & 10 & 43 & 70 & 27 & 0.86 & PCR-RFLP \\
\hline Sayhan $^{14}$ & 2001 & Turkey & Mix & Population-based & Sporadic & 26 & 30 & 11 & 21 & 43 & 12 & 0.20 & PCR-RFLP \\
\hline Hamajima $^{15}$ & 2002 & Japan & Asian & Hospital-based & Sporadic & 58 & 72 & 17 & 91 & 107 & 43 & 0.24 & Allele specific PCR \\
\hline Gemignani $^{16}$ & 2004 & Spain & Caucasian & Hospital-based & Sporadic & 201 & 133 & 18 & 202 & 95 & 19 & 0.09 & Allele specific PCR \\
\hline Schneider $^{17}$ & 2004 & Germany & Caucasian & Population-based & Sporadic & 26 & 26 & 5 & 38 & 41 & 6 & 0.25 & PCR-SSCP \\
\hline Krüger $^{18}$ & 2005 & Germany & Caucasian & Population-based & Hereditary & 180 & 95 & 18 & 150 & 78 & 17 & 0.13 & PCR-RFLP \\
\hline \multirow[t]{2}{*}{ Sotamaa $^{19}$} & 2005 & Finland & Caucasian & Population-based & $\begin{array}{c}\text { Hereditary, } \\
\text { Sporadic }\end{array}$ & 231 & 129 & 19 & 172 & 125 & 26 & 0.62 & PCR-SSCP \\
\hline & & USA & Mix & Population-based & Hereditary & 21 & 7 & 2 & 64 & 41 & 13 & 0.11 & PCR-SSCP \\
\hline Koushik $^{20}$ & 2006 & USA & Mix & Population-based & Sporadic & 228 & 186 & 28 & 498 & 351 & 55 & 0.51 & Allele specific PCR \\
\hline $\operatorname{Lima}^{21}$ & 2006 & Brazil & Mix & Hospital-based & Sporadic & 56 & 38 & 6 & 58 & 36 & 6 & 0.90 & Allele specific PCR \\
\hline Pérez ${ }^{22}$ & 2006 & Argentina & Mix & Population-based & Sporadic & 31 & 20 & 2 & 44 & 53 & 12 & 0.50 & Allele specific PCR \\
\hline Perfumo $^{23}$ & 2006 & Italy & Caucasian & Hospital-based & Sporadic & 28 & 30 & 2 & 90 & 49 & 7 & 0.92 & PCR-RFLP \\
\hline \multirow[t]{2}{*}{ Talseth $^{24}$} & 2006 & Australia & Caucasian & Population-based & Hereditary & 39 & 19 & 3 & 10 & 11 & 0 & 0.10 & Sequencing \\
\hline & & Poland & Caucasian & Population-based & Hereditary & 33 & 19 & 4 & 45 & 28 & 5 & 0.82 & Sequencing \\
\hline $\operatorname{Tan}^{25}$ & 2007 & Germany & Caucasian & Population-based & Sporadic & 312 & 131 & 24 & 343 & 193 & 27 & 0.98 & Allele specific PCR \\
\hline $\mathrm{Zhu}^{26}$ & 2007 & China & Asian & Population-based & Sporadic & 83 & 117 & 85 & 244 & 321 & 105 & 0.97 & PCR-RFLP \\
\hline Grünhage $^{27}$ & 2008 & Germany & Caucasian & Hospital-based & $\begin{array}{c}\text { Hereditary, } \\
\text { Sporadic }\end{array}$ & 105 & 72 & 14 & 123 & 78 & 19 & 0.20 & PCR-RFLP \\
\hline Csejtei $^{28}$ & 2008 & Hungary & Caucasian & Population-based & Sporadic & 66 & 32 & 4 & 62 & 29 & 6 & 0.31 & Allele specific PCR \\
\hline $\mathrm{CaO}^{29}$ & 2009 & Korean & Asian & Population-based & Sporadic & 54 & 67 & 35 & 114 & 140 & 39 & 0.70 & PCR-RFLP \\
\hline Polakova $^{30}$ & 2009 & Germany & Caucasian & Hospital-based & Sporadic & 327 & 225 & 60 & 326 & 237 & 49 & 0.52 & PCR-RFLP \\
\hline Mojtahedi $^{31}$ & 2010 & Iran & Asian & Population-based & Sporadic & 46 & 63 & 23 & 58 & 77 & 28 & 0.78 & Allele specific PCR \\
\hline Aizat $^{32}$ & 2011 & Malaysia & Asian & Hospital-based & Sporadic & 70 & 88 & 44 & 75 & 101 & 25 & 0.31 & PCR-RFLP \\
\hline Dastjerdi $^{33}$ & 2011 & Iran & Asian & Population-based & Sporadic & 97 & 101 & 52 & 76 & 113 & 61 & 0.14 & PCR-RFLP \\
\hline Engin $^{34}$ & 2011 & Turkey & Mix & Hospital-based & Sporadic & 50 & 41 & 5 & 52 & 42 & 14 & 0.24 & PCR-RFLP \\
\hline Joshi $^{35}$ & 2011 & Japan & Asian & Population-based & Sporadic & 239 & 342 & 104 & 310 & 361 & 107 & 0.90 & PCR-RFLP \\
\hline Song $^{36}$ & 2011 & Korea & Asian & $\begin{array}{c}\text { Population- } \\
\text { based }\end{array}$ & Sporadic & 740 & 844 & 244 & 734 & 776 & 190 & 0.48 & TaqMan \\
\hline Zhang $^{37}$ & 2012 & China & Asian & Hospital-based & Sporadic & 147 & 199 & 98 & 196 & 271 & 102 & 0.62 & MALDI-TOF \\
\hline $\mathrm{Oh}^{38}$ & 2014 & Korea & Asian & Hospital-based & Sporadic & 222 & 247 & 76 & 145 & 218 & 65 & 0.25 & PCR-RFLP \\
\hline Singamsetty ${ }^{39}$ & 2014 & India & Asian & Population-based & Sporadic & 16 & 48 & 39 & 37 & 45 & 25 & 0.13 & Sequencing \\
\hline
\end{tabular}

$H W E$, Hardy-Weinberg equilibrium; $P C R$, polymerase chain reaction; RFLP, restriction fragment length polymorphism; MALDI-TOF, Matrix-assisted laser desorption/ionization time-of-flight.

2 studies with overlapping populations $[18,46]$. We also identified 8 studies as eligible [32-39] that were not included in earlier meta-analyses. Thus, our metaanalysis likely provides a more precise estimate of the relationship between the TP53 Arg72Pro polymorphism and CRC risk.

Several studies have indicated that there are multiple differences in the epidemiological, pathological 
Table 2: Meta-analysis of the association between TP53 Arg72Pro polymorphism and colorectal cancer risk

\begin{tabular}{|c|c|c|c|c|c|c|c|c|c|c|c|c|c|}
\hline \multirow[t]{2}{*}{ Subgroup } & \multirow[t]{2}{*}{ NO. } & \multicolumn{3}{|c|}{$C$ vs. $G$} & \multicolumn{3}{|c|}{$C C$ vs. $G G$} & \multicolumn{3}{|c|}{$C C+G C$ vs. $G G$} & \multicolumn{3}{|c|}{$C C$ vs. $G C+G G$} \\
\hline & & OR(95\%CI) & $P_{h}$ & $P_{O R}$ & OR $(95 \% C I)$ & $P_{h}$ & $P_{O R}$ & OR(95\%CI) & $P_{h}$ & $P_{O R}$ & OR(95\%CI) & $P_{h}$ & $P_{O R}$ \\
\hline Overall & 32 & $1.02(0.94-1.10)$ & 0.000 & $0.678^{*}$ & $1.06(0.90-1.25)$ & 0.000 & $0.489^{*}$ & $1.01(0.91-1.11)$ & 0.000 & $0.912^{*}$ & $1.09(0.95-1.24)$ & 0.017 & $0.223^{*}$ \\
\hline \multicolumn{14}{|l|}{ Ethnicity } \\
\hline Caucasian & 12 & $0.96(0.88-1.05)$ & 0.338 & 0.359 & $0.92(0.74-1.15)$ & 0.854 & 0.472 & $0.96(0.86-1.06)$ & 0.130 & 0.399 & $0.94(0.76-1.16)$ & 0.820 & 0.555 \\
\hline Asian & 14 & $1.10(0.98-1.23)$ & 0.000 & $0.102^{*}$ & $1.25(0.99-1.58)$ & 0.000 & $0.060^{*}$ & $1.08(0.93-1.26)$ & 0.000 & $0.300^{*}$ & $1.22(1.02-1.45)$ & $0.005^{*}$ & $0.026^{*}$ \\
\hline Mixed & 6 & $0.94(0.82-1.09)$ & 0.056 & 0.416 & $0.79(0.55-1.12)$ & 0.244 & 0.181 & $0.96(0.81-1.15)$ & 0.057 & 0.663 & $0.82(0.58-1.16)$ & 0.385 & 0.261 \\
\hline \multicolumn{14}{|l|}{ Source of controls } \\
\hline $\begin{array}{l}\text { Population- } \\
\text { based }\end{array}$ & 20 & $1.01(0.90-1.14)$ & 0.000 & $0.825^{*}$ & $1.12(0.89-1.41)$ & 0.000 & $0.319^{*}$ & $0.99(0.85-1.14)$ & 0.000 & $0.843^{*}$ & $1.15(0.97-1.36)$ & 0.046 & $0.102^{*}$ \\
\hline $\begin{array}{l}\text { Hospital- } \\
\text { based }\end{array}$ & 12 & $1.01(0.94-1.09)$ & 0.155 & 0.744 & $1.00(0.85-1.19)$ & 0.165 & 0.974 & $1.04(0.98-1.10)$ & 0.251 & 0.900 & $1.04(0.89-1.21)$ & 0.093 & 0.636 \\
\hline \multicolumn{14}{|l|}{ Tumor location } \\
\hline$\underline{\text { Colon cancer }}$ & 8 & $1.12(0.96-1.32)$ & 0.020 & $0.159^{*}$ & $1.23(0.88-1.73)$ & 0.041 & $0.228^{*}$ & $1.21(0.94-1.56)$ & 0.005 & $0.145^{*}$ & $1.14(0.94-1.39)$ & 0.421 & 0.185 \\
\hline (Caucasian) & 2 & $1.09(0.90-1.32)$ & 0.456 & 0.365 & $1.19(0.77-1.84)$ & 0.160 & 0.432 & $1.11(0.87-1.41)$ & 0.942 & 0.410 & $1.14(0.75-1.73)$ & 0.104 & 0.551 \\
\hline (Asian) & 5 & $1.16(0.86-1.55)$ & 0.003 & $0.332^{*}$ & $1.35(0.79-2.30)$ & 0.015 & $0.275^{*}$ & $1.29(0.78-2.13)$ & 0.001 & $0.319^{*}$ & $1.18(0.93-1.50)$ & 0.414 & 0.174 \\
\hline (Mixed) & 1 & $1.15(0.93-1.41)$ & - & 0.192 & $1.07(0.61-1.88)$ & - & 0.808 & $1.25(0.97-1.62)$ & - & 0.091 & $0.96(0.56-1.66)$ & - & 0.888 \\
\hline 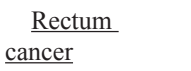 & 8 & $1.13(0.92-1.38)$ & 0.001 & $0.257^{*}$ & $1.36(0.93-1.99)$ & 0.010 & $0.108^{*}$ & $1.07(0.83-1.36)$ & 0.018 & $0.615^{*}$ & $1.34(1.12-1.62)$ & 0.125 & 0.002 \\
\hline (Caucasian) & 2 & $0.90(0.72-1.13)$ & 0.549 & 0.359 & $1.00(0.61-1.65)$ & 0.581 & 0.998 & $0.82(0.62-1.09)$ & 0.549 & 0.161 & $1.11(0.68-1.81)$ & 0.687 & 0.671 \\
\hline (Asian) & 5 & $1.24(0.93-1.67)$ & 0.001 & $0.142^{*}$ & $1.53(0.88-2.66)$ & 0.002 & $0.128^{*}$ & $1.24(0.85-1.79)$ & 0.010 & $0.264^{*}$ & $1.41(0.97-2.05)$ & 0.034 & $0.071^{*}$ \\
\hline (Mixed) & 1 & $1.09(0.78-1.53)$ & - & 0.626 & $1.42(0.64-3.15)$ & - & 0.387 & $1.03(0.68-1.58)$ & - & 0.877 & $1.44(0.66-3.11)$ & - & 0.360 \\
\hline \multicolumn{14}{|l|}{ Type of CRC } \\
\hline$\underline{\text { Sporadic }}$ & 28 & $1.03(0.95-1.12)$ & 0.000 & $0.459^{*}$ & $1.09(0.92-1.29)$ & 0.000 & $0.323^{*}$ & $1.02(0.92-1.14)$ & 0.000 & $0.695^{*}$ & $1.11(0.97-1.27)$ & 0.018 & $0.122^{*}$ \\
\hline (Caucasian) & 9 & $0.97(0.88-1.07)$ & 0.069 & 0.594 & $0.97(0.76-1.24)$ & 0.904 & 0.803 & $0.96(0.85-1.09)$ & 0.077 & 0.540 & $0.99(0.78-1.25)$ & 0.838 & 0.928 \\
\hline (Asian) & 14 & $1.10(0.98-1.23)$ & 0.000 & $0.102^{*}$ & $1.25(0.99-1.58)$ & 0.000 & $0.060^{*}$ & $1.08(0.93-1.26)$ & 0.000 & $0.300^{*}$ & $1.22(1.02-1.45)$ & $0.005^{*}$ & $0.026^{*}$ \\
\hline (Mixed) & 5 & $0.97(0.84-1.11)$ & 0.299 & 0.072 & $0.81(0.56-1.17)$ & 0.186 & 0.262 & $0.99(0.83-1.19)$ & 0.075 & 0.923 & $0.84(0.59-1.19)$ & 0.287 & 0.328 \\
\hline Hereditary & 6 & $0.86(0.73-1.01)$ & 0.422 & 0.072 & $0.69(0.45-1.04)$ & 0.374 & 0.078 & $0.87(0.71-1.06)$ & 0.465 & 0.158 & $0.71(0.47-1.07)$ & 0.417 & 0.106 \\
\hline (Caucasian) & 5 & $0.88(0.75-1.05)$ & 0.474 & 0.148 & $0.71(0.46-1.10)$ & 0.284 & 0.124 & $0.89(0.73-1.10)$ & 0.551 & 0.290 & $0.73(0.48-1.11)$ & 0.300 & 0.141 \\
\hline (Mixed) & 1 & $0.57(0.28-1.16)$ & - & 0.118 & $0.47(0.10-2.25)$ & - & 0.344 & $0.51(0.22-1.20)$ & - & 0.123 & $0.58(0.12-2.71)$ & - & 0.486 \\
\hline \multicolumn{14}{|l|}{ Genotype methods } \\
\hline PCR-RFLP & 14 & $1.04(0.92-1.18)$ & 0.000 & $0.519^{*}$ & $1.07(0.81-1.40)$ & 0.000 & $0.634^{*}$ & $1.04(0.88-1.23)$ & 0.001 & $0.628^{*}$ & $1.10(0.89-1.36)$ & 0.011 & $0.381^{*}$ \\
\hline $\begin{array}{l}\text { Allele specific } \\
\text { PCR }\end{array}$ & 10 & $0.98(0.89-1.07)$ & 0.153 & 0.636 & $0.93(0.74-1.16)$ & 0.453 & 0.518 & $0.98(0.88-1.11)$ & 0.119 & 0.791 & $0.94(0.76-1.169)$ & 0.348 & 0.543 \\
\hline PCR-SSCP & 3 & $0.77(0.62-0.95)$ & 0.383 & 0.013 & $0.61(0.36-1.03)$ & 0.510 & 0.065 & $0.74(0.57-0.95)$ & 0.514 & 0.020 & $0.68(0.41-1.14)$ & 0.559 & 0.142 \\
\hline Sequencing & 3 & $1.20(0.65-2.22)$ & 0.031 & $0.554^{*}$ & $2.66(1.39-5.08)$ & 0.328 & 0.003 & $1.18(0.44-3.12)$ & 0.009 & $0.745^{*}$ & $1.85(1.08-3.16)$ & 0.731 & 0.025 \\
\hline
\end{tabular}

OR odds ratio; $95 \%$ CI 95\% confidence interval; $P_{O R}$, pool $P$ value; $P_{h}, P$ value of heterogeneity test;

* Estimates for random-effects model; otherwise, fixed-effects model was used.

and molecular features of CRCs [56-58]. Kapiteijn et al. indicated that rectal cancer may involve more nuclear $\beta$-catenin in the APC $/ \beta$-catenin pathway than colon cancer and reported that the p53-pathway also appears to be more important in rectal cancer [57]. In another study,
Slattery et al. found that rectal and distal colon tumors are more likely to have a p53 mutation than proximal colon tumors [58]. When we stratified based on tumor location, we observed a significant association between the TP53 Arg72Pro CC genotype and rectal cancer, but no 
association was observed between this genotype and colon cancer. One possible explanation for this finding could be that different bacterial flora and a longer transit time in the rectum might change the contact between intestinal cells and potential carcinogens or promoters in the fecal stream, which may lead to more (exogenous) mutations of p53.

Factors known to affect the risk of CRC include gender, age, environmental factors and chronic
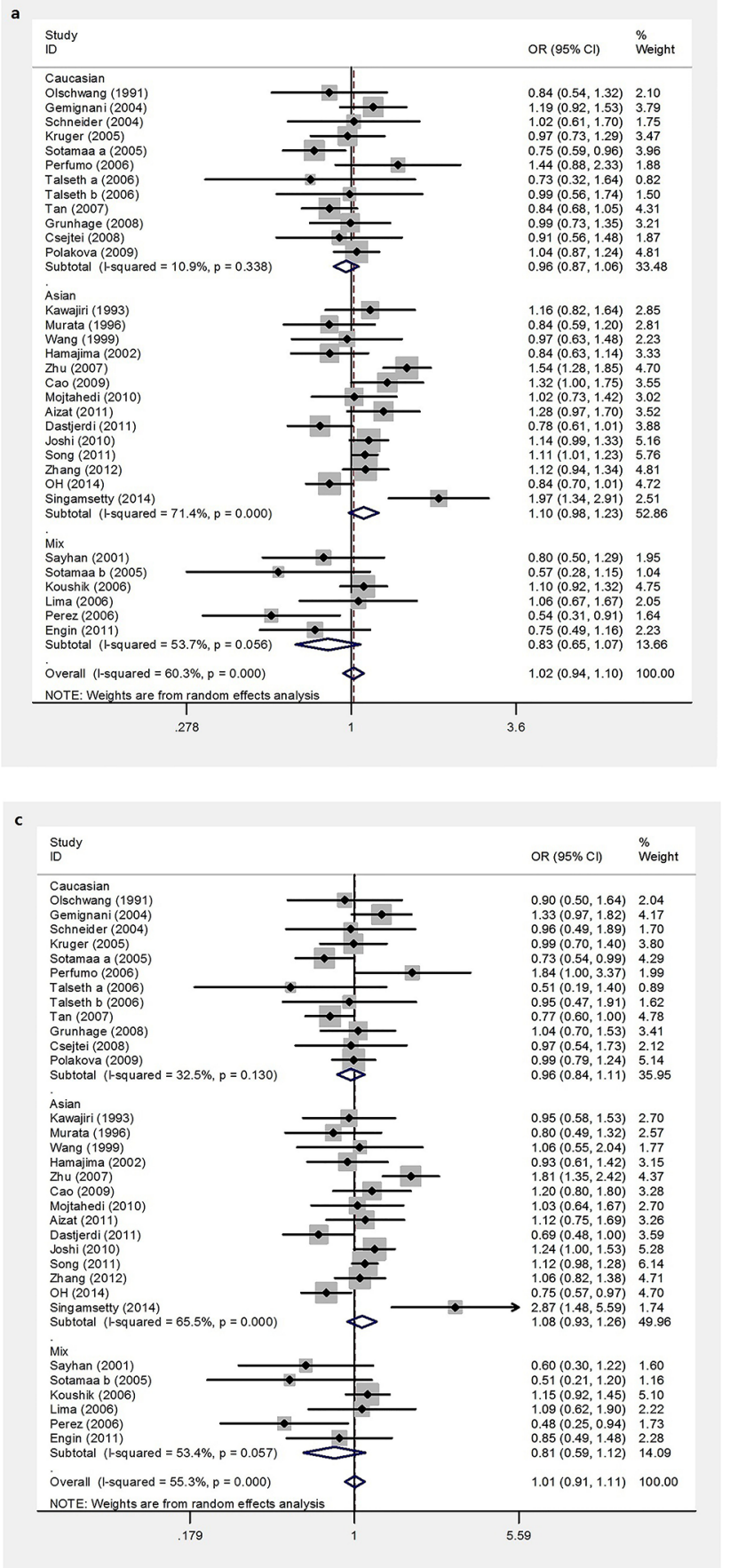

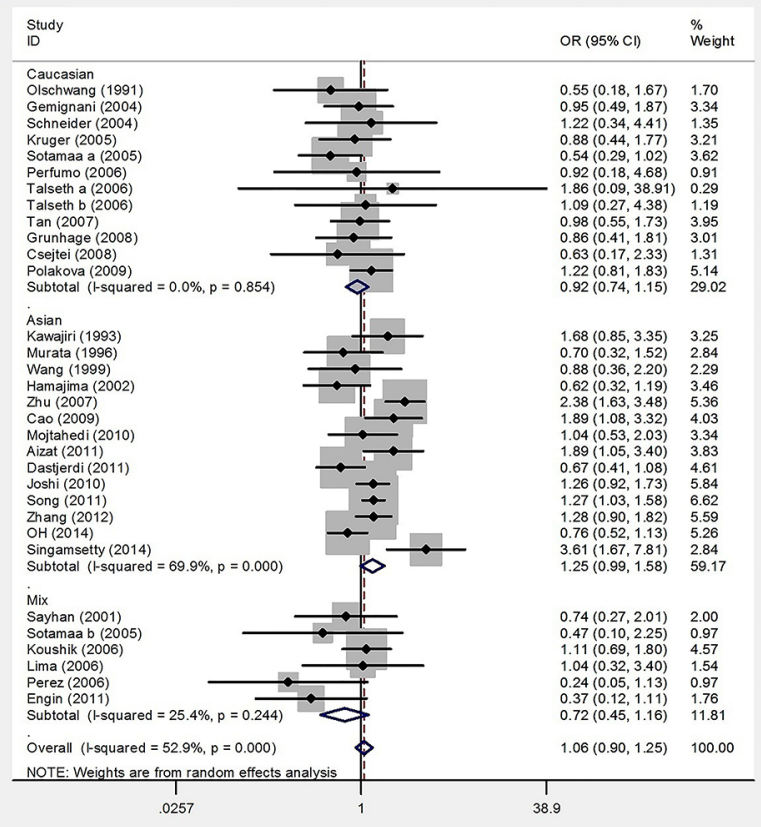

d

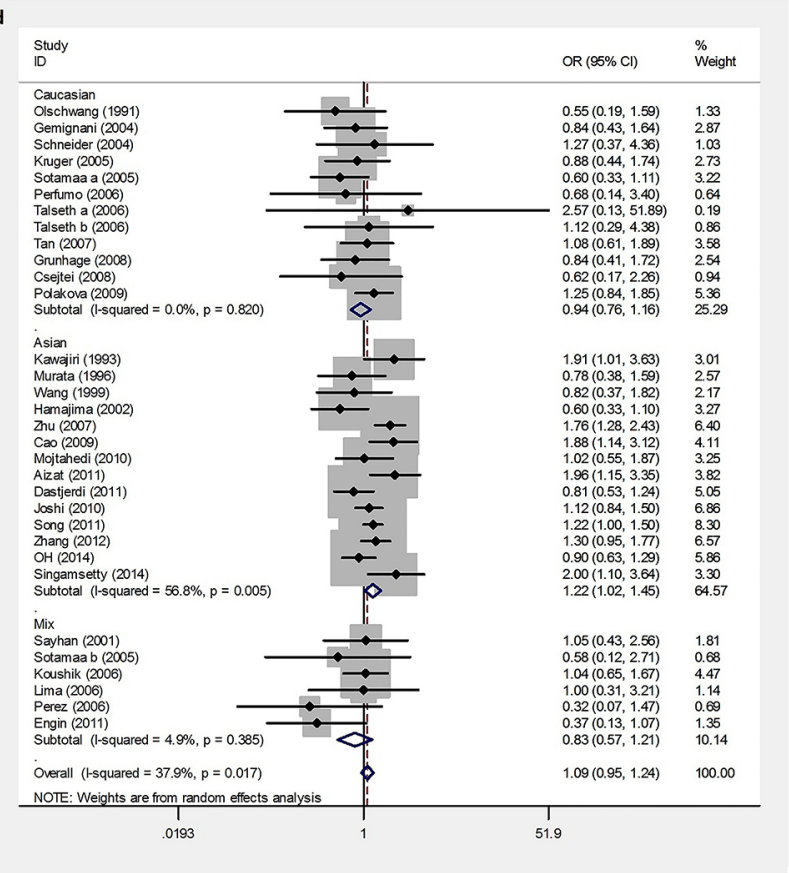

Figure 2: Forest plots of TP53 Arg72Pro polymorphism and CRC risk. a. allele model, b. homozygous model, c. dominant models, d. recessive models. 
inflammation. Joshi et al. found that men with the CC genotype and $\mathrm{C}$ allele had significantly higher risk for CRC than women with the same genotype [35]. Aizat et al. found that carriers of CC genotype aged 50 years and older were also at significantly greater risk for CRC [32]. However, no significant associations were found between these two confounding factors and CRC susceptibility in other studies [26, 29]. The difference may be explained by differences in the groups studied or populations and/ or by differences in environmental exposure and lifestyle factors. Additional studies with a large patient cohort are needed to verify these initial observations.

Our meta-analysis had several limitations. First, we did not calculate an adjusted estimate for the association between the TP53 Arg72Pro polymorphism and CRC risk because not all studies reported adjusted ORs.
Second, because heterogeneity was obvious, even in some sub-analyses, other potential confounding factors appeared to be present in the included studies; we did not take these confounding factors into account. Third, due to an absence of information, we were unable to assess other factors such as gender, age, alcohol consumption and smoking status, which may have modified the association. Finally, potential gene-gene and geneenvironment interactions were not analyzed due to a lack of relevant data.

In summary, our updated meta-analysis demonstrated that the TP53 Arg72Pro polymorphism CC genotype may contribute to an increased risk of $\mathrm{CRC}$, especially for rectal cancer and among Asians. Future well-designed studies with larger samples are needed to confirm our findings.

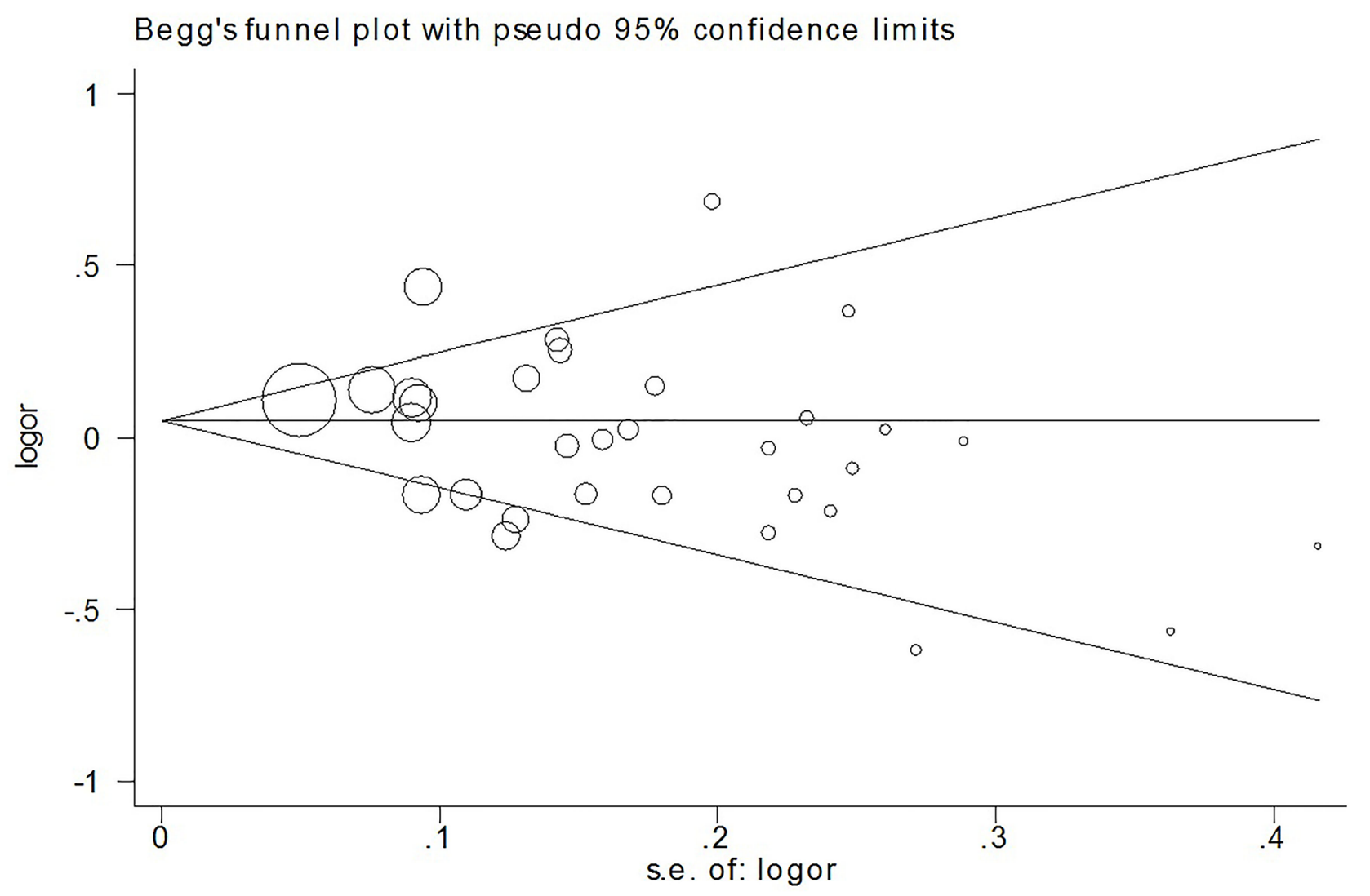

Figure 3: Beggar's funnel plot of TP53 Arg72Pro polymorphism and CRC risk under the allele model. 


\section{MATERIALS AND METHODS}

\section{Identification of eligible studies}

Potentially relevant articles published prior to December 2014 were identified in the PubMed, EMBASE, Web of Knowledge, and Chinese National Knowledge Infrastructure databases using the following key words: "TP53 or P53," "polymorphism or variant," and "colorectal cancer, colon or CRC." Additional studies on the topic of interest were identified by hand-searching the reference lists of the retrieved articles. When multiple publications reported on the same or overlapping data, the most recent study with the largest sample size was selected.

\section{Inclusion and exclusion criteria}

The studies included in our meta-analysis were required to meet the following criteria: 1 ) the study was a case-control or cohort study; 2) the study investigated the association between the TP53 Arg72Pro polymorphism and $\mathrm{CRC}$ risk; 3) the study provided sufficient information to estimate ORs and 95\% CIs; and 4) the study had a control genotype distribution in HWE. Studies were excluded for the following reasons: 1) the study was not a case-control study; 2) the publication contained incomplete data; and 3) the study was a duplicate of a previous publication.

\section{Data extraction}

Data were independently extracted by two reviewers (Dai and Sun) using a standardized data extraction form. Disagreements were resolved through discussion. The extracted data included the following items: first author, publication year, country of origin, ethnicity, source of control, sample sizes, genotype distribution in cases and controls, $P$-value for HWE, and genotyping methods.

\section{Statistical analysis}

Pooled ORs with corresponding 95\% CIs were used to evaluate the strength of the observed associations. Four genetic contrast models, including allelic contrast (C vs. G), homozygote comparisons (CC vs. GG), dominant models $(\mathrm{CC}+\mathrm{GC}$ vs. $\mathrm{GG})$, and recessive models (CC vs. $\mathrm{GC}+\mathrm{GG})$, were applied. HWE was evaluated in the control group for each study using the $\chi^{2}$ test, and the significance level was set at $P<0.05$. Between-study heterogeneity was assessed by calculating the $Q$-statistic and quantified using the $I^{2}$ value. A fixed effect model that used the Mantel-Haenszel approach was applied to calculate the pooled ORs if the between-study heterogeneity was not significant [59]. A random effect model that used DerSimonian and Laird's method was adopted when the between-study heterogeneity was obvious [60]. When the Q test $P>0.05$ and $I^{2}<50 \%$, the fixed-effects model was used; otherwise, the random-effects model was used. Subgroup analyses were performed based on ethnicity, source of controls, tumor location and genotype method. Sensitivity analysis was performed to determine the influence of single datasets on the combined estimates. Begg's funnel plot and Egger's test were used to assess publication bias [61, 62]. All analyses were performed using Stata software version 12.0 (Stata Corp., College Station, TX), and all $P$ values were two-sided.

\section{ACKNOWLEDGMENTS AND FUNDING}

This study was supported by grants from the National Natural Science Foundation of China (No. 81573654).

\section{CONFLICTS OF INTEREST}

The authors indicated no financial relationships.

\section{REFERENCES}

1. Siegel R, Naishadham D, Jemal A. Cancer statistics, 2013. Cancer J Clin. 2013;63:11-30.

2. Jemal A, Bray F, Center MM, Ferlay J, Ward E, Forman D. Global cancer statistics. Cancer J Clin. 2011;61:69-90.

3. Sung JJ, Lau JY, Goh KL, Leung WK; Asia Pacific Working Group on Colorectal Cancer. Increasing incidence of colorectal cancer in Asia: implications for screening. Lancet Oncol. 2005;6:871-6.

4. Kim HJ, Yu MH, Kim H, Byun J, Lee C. Noninvasive molecular biomarkers for the detection of colorectal cancer. BMB Rep. 2008;41:685-692.

5. Cheah PY. Recent advances in colorectal cancer genetics and diagnostics. Crit Rev Oncol Hematol. 2009;69:45-55.

6. Tsui IF, Poh CF, Garnis C, Rosin MP, Zhang L, Lam WL. Multiple pathways in the FGF signaling network are frequently deregulated by gene amplification in oral dysplasias. Int J Cancer. 2009;125:2219-28.

7. Berchuck A, Kohler MF, Marks JR, Wiseman R, Boyd J, Bast RC Jr. The p53 tumor suppressor gene frequently is altered in gynecologic cancers. Am J Obstet Gynecol. 1994; 170:246-52.

8. Francisco G, Menezes PR, Eluf-Neto J, Chammas R. Arg72Pro TP53 polymorphism and cancer susceptibility: a comprehensive meta-analysis of 302 case-control studies. Int J Cancer. 2011;129:920-30.

9. Pim D, Banks L. p53 polymorphic variants at codon 72 exert different effects on cell cycle progression. Int $\mathrm{J}$ Cancer. 2004;108:196-9.

10. Olschwang S, Laurent-Puig P, Vassal A, Salmon RJ, Thomas G. Characterization of a frequent polymorphism in the coding sequence of the Tp53 gene in colonic cancer patients and a control population. Hum Genet. 1991;86:369-70. 
11. Kawajiri K, Nakachi K, Imai K, Watanabe J, Hayashi $\mathrm{S}$. Germ line polymorphisms of $\mathrm{p} 53$ and CYP1A1 genes involved in human lung cancer. Carcinogenesis. 1993;14:1085-9.

12. Murata M, Tagawa M, Kimura M, Kimura H, Watanabe S, Saisho H. Analysis of a germ line polymorphism of the p53 gene in lung cancer patients; discrete results with smoking history. Carcinogenesis. 1996;17:261-4.

13. Wang NM, Tsai CH, Yeh KT, Chen SJ, Chang JG. P53 codon $72 \mathrm{Arg}$ polymorphism is not a risk factor for carcinogenesis in the Chinese. Int J Mol Med. 1999;4:249-52.

14. Sayhan N, Yazici H, Budak M, Bitisik O, Dalay N. P53 codon 72 genotypes in colon cancer. Association with human papillomavirus infection. Res Commun Mol Pathol Pharmacol. 2001;109:25-34.

15. Hamajima N, Matsuo K, Suzuki T, Nakamura T, Matsuura A, Hatooka S, Shinoda M, Kodera Y, Yamamura Y, Hirai T, Kato T, Tajima K. No associations of p73 G4C14-toA4T14 at exon 2 and p53 Arg72Pro polymorphisms with the risk of digestive tract cancers in Japanese. Cancer Lett. 2002;181:81-5.

16. Gemignani F, Moreno V, Landi S, Moullan N, Chabrier A, Gutiérrez-Enríquez S, Hall J, Guino E, Peinado MA, Capella G, Canzian F. A TP53 polymorphism is associated with increased risk of colorectal cancer and with reduced levels of TP53 mRNA. Oncogene. 2004;23:1954-6.

17. Schneider-Stock R, Boltze C, Peters B, Szibor R, Landt O, Meyer F, Roessner A. Selective loss of codon 72 proline p53 and frequent mutational inactivation of the retained arginine allele in colorectal cancer. Neoplasia. 2004;6:529-35.

18. KrügerS,BierA,EngelC,MangoldE,PagenstecherC, vonKnebel Doeberitz M, Holinski-Feder E, Moeslein G, Schulmann K, PlaschkeJ,RüschoffJ, SchackertHK; German Hereditary NonPolyposis Colorectal Cancer Consortium. The p53 codon 72 variation is associated with the age of onset of hereditary non-polyposis colorectal cancer (HNPCC). J Med Genet. 2005;42:769-73.

19. Sotamaa K, Liyanarachchi S, Mecklin JP, Järvinen $H$, Aaltonen LA, Peltomäki P, de la Chapelle A. p53 codon 72 and MDM2 SNP309 polymorphisms and age of colorectal cancer onset in Lynch syndrome. Clin Cancer Res. 2005;11:6840-4.

20. Koushik A, Tranah GJ, Ma J, Stampfer MJ, Sesso HD, Fuchs CS, Giovannucci EL, Hunter DJ. p53 Arg72Pro polymorphism and risk of colorectal adenoma and cancer. Int J Cancer. 2006;119:1863-8.

21. Lima JM, Serafim PV, Silva ID, Forones NM. Role of the genetic polymorphism of $\mathrm{p} 53$ (codon 72) gene in colorectal cancer. Arq Gastroenterol. 2006;43:8-13.

22. Pérez LO, Abba MC, Dulout FN, Golijow CD. Evaluation of p53 codon 72 polymorphism in adenocarcinomas of the colon and rectum in La Plata, Argentina. World J Gastroenterol. 2006;12:1426-9.

23. Perfumo C, Bonelli L, Menichini P, Inga A, Gismondi V, Ciferri E, Percivale P, Bianchi Scarrà G, Nasti S, Fronza
G, Varesco L. Increased risk of colorectal adenomas in Italian subjects carrying the p53 PIN3 A2-Pro72 haplotype. Digestion. 2006;74:228-35.

24. Talseth BA, Meldrum C, Suchy J, Kurzawski G, Lubinski J, Scott RJ. MDM2 SNP309 T>G alone or in combination with the TP53 R72P polymorphism does not appear to influence disease expression and age of diagnosis of colorectal cancer in HNPCC patients. Int J Cancer. 2007;120:563-5.

25. Tan XL, Nieters A, Hoffmeister M, Beckmann L, Brenner H, Chang-Claude J. Genetic polymorphisms in TP53, nonsteroidal anti-inflammatory drugs and the risk of colorectal cancer: evidence for gene-environment interaction? Pharmacogenet Genomics. 2007;17:639-45.

26. Zhu ZZ, Wang AZ, Jia HR, Jin XX, He XL, Hou LF, Zhu G. Association of the TP53 codon 72 polymorphism with colorectal cancer in a Chinese population. Jpn J Clin Oncol. 2007;37:385-90.

27. Grünhage F, Jungck M, Lamberti C, Berg C, Becker U, Schulte-Witte H, Plassmann D, Rahner N, Aretz S, Friedrichs N, Buettner R, Sauerbruch T, Lammert F. Association of familial colorectal cancer with variants in the E-cadherin (CDH1) and cyclin D1 (CCND1) genes. Int J Colorectal Dis. 2008;32:147-54.

28. Csejtei A, Tibold A, Varga Z, Koltai K, Ember A, Orsos Z, Feher G, Horvath OP, Ember I, Kiss I. GSTM, GSTT and p53 polymorphisms as modifiers of clinical outcome in colorectal cancer. Anticancer Res. 2008;28:1917-22.

29. Cao Z, Song JH, Park YK, Maeng EJ, Nam SW, Lee JY, Park WS. The p53 codon 72 polymorphism and susceptibility to colorectal cancer in Korean patients. Neoplasma. 2009;56:114-8.

30. Polakova V, Pardini B, Naccarati A, Landi S, Slyskova J, Novotny J, Vodickova L, Bermejo JL, Hanova M, Smerhovsky Z, Tulupova E, Kumar R, Hemminki K, et al. Genotype and haplotype analysis of cell cycle genes in sporadic colorectal cancer in the Czech Republic. Hum Mutat. 2009;30:661-8.

31. Mojtahedi Z, Haghshenas MR, Hosseini SV, Fattahi MJ, Ghaderi A. p53 codon 72 polymorphism in stomach and colorectal adenocarcinomas in Iranian patients. Indian $\mathbf{J}$ Cancer. 2010;47:31-4.

32. Aizat AA, Shahpudin SN, Mustapha MA, Zakaria Z, Sidek AS, Abu Hassan MR, Ankathil R. Association of Arg72Pro of P53 polymorphism with colorectal cancer susceptibility risk in Malaysian population. Asian Pac J Cancer Prev. 2011;12:2909-13.

33. Dastjerdi MN. TP53 codon 72 polymorphism and P53 protein expression in colorectal cancer specimens in Isfahan. Acta Med Iran. 2011;49:71-7.

34. Engin AB, Karahalil B, Karakaya AE, Engin A. Association between XRCC1 ARG399GLN and P53 ARG72PRO polymorphisms and the risk of gastric and colorectal cancer in Turkish population. Arh Hig Rada Toksikol. 2011;62:207-14. 
35. Joshi AM, Budhathoki S, Ohnaka K, Mibu R, Tanaka M, Kakeji Y, Maehara Y, Okamura T, Ikejiri K, Futami K, Maekawa T, Yasunami Y. TP53 R72P and MDM2 SNP309 polymorphisms and colorectal cancer risk: the Fukuoka Colorectal Cancer Study. Jpn J Clin Oncol. 2011;41:232-8.

36. Song HR, Kweon SS, Kim HN, Piao JM, Yun WJ, Choi JS, Hwang JE, Yoon JY, Kim HR, Park YK, Kim SH, Choi YD, Shin MH. p53 codon 72 polymorphism in patients with gastric and colorectal cancer in a Korean population. Gastric Cancer. 2011;14:242-8.

37. Zhang Y, Liu L, Tang Y, Chen C, Wang Q, Xu J, Yang C, Miao X, Wei S, Chen J, Nie S. Polymorphisms in TP53 and MDM2 contribute to higher risk of colorectal cancer in Chinese population: a hospital-based, case-control study. Mol Biol Rep. 2012;39:9661-8.

38. Oh J, Kim JW, Lee BE, Jang MJ, Chong SY, Park PW, Hwang SG, Oh D, Kim NK. Polymorphisms of the pri-miR34b/c promoter and TP53 codon 72 are associated with risk of colorectal cancer. Oncol Rep. 2014;31:995-1002.

39. Singamsetty GK, Malempati S, Bhogadhi S, Kondreddy R, Govatati S, Tangudu NK, Govatati S, kuraganti AK, Bhanoori M, Kassetty K. TP53 alterations and colorectal cancer predisposition in south Indian population: a casecontrol study. Tumor Biol. 2014;35:2303-11.

40. Langerød A, Bukholm IR, Bregård A, Lønning PE, Andersen TI, Rognum TO, Meling GI, Lothe RA, BørresenDale AL. The TP53 codon 72 polymorphism may affect the function of TP53 mutations in breast carcinomas but not in colorectal carcinomas. Cancer Epidemiol Biomarkers Prev. 2002;11:1684-8.

41. Jones JS, Chi X, Gu X, Lynch PM, Amos CI, Frazier ML. p53 polymorphism and age of onset of hereditary nonpolyposis colorectal cancer in a Caucasian population. Clin Cancer Res. 2004;10:5845-9.

42. Lung FW, Lee TM, Shu BC, Chang FH. p53 codon 72 polymorphism and susceptibility malignancy of colorectal cancer in Taiwan. J Cancer Res Clin Oncol. 2004;130:728-32.

43. Talseth BA, Meldrum C, Suchy J, Kurzawski G, Lubinski J, Scott RJ. Age of diagnosis of colorectal cancer in HNPCC patients is more complex than that predicted by $\mathrm{R} 72 \mathrm{P}$ polymorphism in TP53. Int J Cancer. 2006;118:2479-84.

44. Khan SA, Idrees K, Forslund A, Zeng Z, Rosenberg S, Pincas H, Barany F, Offit K, Laquaglia MP, Paty PB. Genetic variants in germline TP53 and MDM2 SNP309 are not associated with early onset colorectal cancer. J Surg Oncol. 2008;97:621-5.

45. Katkoori VR, Jia X, Shanmugam C, Wan W, Meleth $\mathrm{S}$, Bumpers H, Grizzle WE, Manne U. Prognostic significance of p53 codon 72 polymorphism differs with race in colorectal adenocarcinoma. Clin Cancer Res. 2009;15:2406-16.

46. Krüger S, Engel C, Bier A, Silber AS, Görgens H, Mangold E, Pagenstecher C, Holinski-Feder E, von Knebel Doeberitz M, Royer-Pokora B, Dechant S, Pox C, Rahner N, et al. The additive effect of p53 Arg72Pro and RNASEL Arg462Gln genotypes on age of disease onset in Lynch syndrome patients with pathogenic germline mutations in $\mathrm{MSH} 2$ or MLH1. Cancer Lett. 2007;252:55-64.

47. Själander A, Birgander R, Athlin L, Stenling R, Rutegård J, Beckman L, Beckman G. P53 germ line haplotypes associated with increased risk for colorectal cancer. Carcinogenesis. 1995;16:1461-4.

48. Dakouras A, Nikiteas N, Papadakis E, Perakis M, Valis D, Rallis G, Tzanakis N, Peros G, Tsigkris C, Kittas C, Karakitsos P. P53Arg72 homozygosity and its increased incidence in left-sided sporadic colorectal adenocarcinomas, in a Greek-Caucasian population. Anticancer Res. 2008;28:1039-43.

49. Sameer AS, Shah ZA, Syeed N, Banday MZ, Bashir SM, Bhat BA, Siddiqi MA. TP53 Pro47Ser and Arg72Pro polymorphisms and colorectal cancer predisposition in an ethnic Kashmiri population. Genet Mol Res. 2010;9:651-60.

50. Lichtenstein P, Holm NV, Verkasalo PK, Iliadou A, Kaprio J, Koskenvuo M, Pukkala E, Skytthe A, Hemminki K. Environmental and heritable factors in the causation of cancer--analyses of cohorts of twins from Sweden, Denmark, and Finland. N Engl J Med. 2000;343:78-85

51. Dumont P, Leu JI, Della Pietra AC 3rd, George DL, Murphy M. The codon 72 polymorphic variants of p53 have markedly different apoptotic potential. Nat Genet. 2003;33:357-65.

52. Thomas M, Kalita A, Labrecque S, Pim D, Banks L, Matlashewski G. Two polymorphic variants of wild-type p53 differ biochemically and biologically. Mol Cell Biol. 1999;19:1092-100.

53. Boyd SD, Tsai KY, Jacks T. An intact HDM2 RING-finger domain is required for nuclear exclusion of p53. Nat Cell Biol. 2000;2:563-8.

54. Tang NP, Wu YM, Wang B, Ma J. Systematic review and meta-analysis of the association between P53 codon 72 polymorphism and colorectal cancer. Eur J Surg Oncol. 2010;36:431-8.

55. Wang JJ, Zheng Y, Sun L, Wang L, Yu PB, Dong JH, Zhang L, Xu J, Shi W, Ren YC. TP53 codon 72 polymorphism and colorectal cancer susceptibility: a meta-analysis. Mol Biol Rep. 2011;38:4847-53.

56. Brändstedt J, Wangefjord S, Nodin B, Gaber A, Manjer J, Jirström K. Gender, anthropometric factors and risk of colorectal cancer with particular reference to tumour location and TNM stage: a cohort study. Biol Sex Differ. 2012;3:23.

57. Kapiteijn E, Liefers GJ, Los LC, Kranenbarg EK, Hermans J, Tollenaar RA, Moriya Y, van de Velde CJ, van Krieken JH. Mechanisms of oncogenesis in colon versus rectal cancer. J Pathol. 2001;195:171-8.

58. Slattery ML, Curtin K, Wolff RK, Boucher KM, Sweeney C, Edwards S, Caan BJ, Samowitz W. A comparison of colon and rectal somatic DNA alterations. Dis Colon Rectum. 2009;52:1304-11. 
59. Mantel N, Haenszel W. Statistical aspects of the analysis of data from retrospective studies of disease. J Natl Cancer Inst. 1959;22:719-48.

60. DerSimonian R, Laird N. Meta-analysis in clinical trials. Control Clin Trials. 1986;7:177-88.

61. Egger M, Davey Smith G, Schneider M, Minder C. Bias in meta-analysis detected by a simple, graphical test. BMJ. 1997;315:629-34.
62. Stuck AE, Rubenstein LZ, Wieland D. Bias in meta-analysis detected by a simple, graphical test. Asymmetry detected in funnel plot was probably due to true heterogeneity. BMJ. 1998;316:469-70. 